Supporting Information for

\title{
Narjatamolide, an Unusual Homo-guaiane Sesquiterpene \\ Lactone from Nardostachys jatamansi
}

Tian Chai, ${ }^{\#}$ Xian-Hua Meng,, Cheng-Bo Wang, Kai Wang, Li-Mei Ma, Yan-Ping Shi, Jun-Li Yang*

Corresponding Author

* Tel: +86-931-4968385. Fax: +86-931-4968385. E-mail: yangj1@licp.cas.cn (J.-L. Yang). 


\section{Content}

\section{EXPERIMENTAL SECTION}

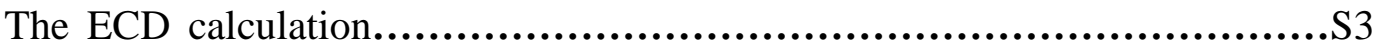

Figure S1. The effect of 1 in different concentrations on morphology of BEL-7402 cells under microscope.

Figure S2. (A) The effect of $\mathbf{1}$ on cell cycle. (B) Compound $\mathbf{1}$ dose dependently induced G2/M phase arrest of BEL-7402 cells.

Figure S3. PBE1PBE/TZVP optimized lowest energy 3D conformer of 1............S4

Table S1. The Cartesian coordinates for the lowest-energy conformer of $\mathbf{1}$ in the ECD calculations

Table S2. The single point energy for the lowest-energy conformer of $\mathbf{1}$ in the ECD calculations S5

Table S3. The imaginary frequencies for the lowest-energy conformer of 1......... S5

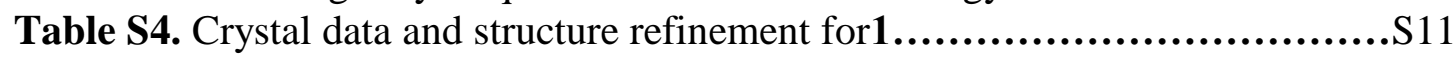

Figure S4. HRESIMS Spectrum of 1.......................................... 13

Figure S5. IR Spectrum of 1.................................................. 14

Figure S6. UV Spectrum of $\mathbf{1}(\mathrm{MeOH})$.......................................... 14

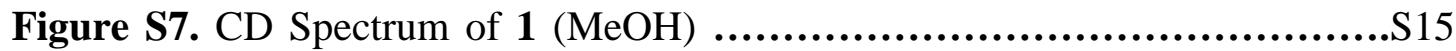

Figure S8. ${ }^{1} \mathrm{H}$ NMR Spectrum of $1\left(400 \mathrm{MHz}, \mathrm{CDCl}_{3}\right)$.........................S15

Figure S9. ${ }^{13} \mathrm{C}$ NMR Spectrum of $\mathbf{1}\left(100 \mathrm{MHz}, \mathrm{CDCl}_{3}\right) \ldots \ldots \ldots \ldots \ldots \ldots \ldots . . . . . . . . . . . . . . .616$

Figure S10. ${ }^{1} \mathrm{H}^{-1}{ }^{1} \mathrm{C}$ COSY Spectrum of $1\left(400 \mathrm{MHz}, \mathrm{CDCl}_{3}\right)$......................... 16

Figure S11. HSQC Spectrum of 1 (400 MHz for ${ }^{1} \mathrm{H}$ NMR, $\left.\mathrm{CDCl}_{3}\right)$.................S17

Figure S12. HMBC Spectrum of 1 (400 MHz for ${ }^{1} \mathrm{H}$ NMR, $\left.\mathrm{CDCl}_{3}\right)$..............S17

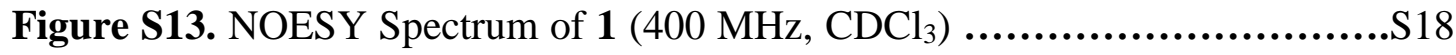

Figure S14. Melting point of 1................................................... 18 


\section{EXPERIMENTAL SECTION}

\section{The ECD calculation}

ECD calculation was determined by quantum chemical TDDFT calculations of using Gaussian 16 series of programs.

Firstly, conformational analyses were carried out via molclus software, ${ }^{1}$ and then using xtb software (GFN1-xTB) ${ }^{2}$ for initial optimization and removal of energy overlap. In the energy window of $2 \mathrm{Kcal} / \mathrm{mol}$, the results showed the lowest-energy conformers for compound 1 (Figure S1). The conformer was then re-optimized using TDDFT at the B3LYP/def2tzvp level in the Gaussian 16 program, and the vibrational frequencies analysis is performed at the same level to ensure their stability and none of imaginary frequencies. ECD is calculated at the PBE1PBE/TZVP level. In order to calculate the result more accurately, nstates is set to 20 . And in order to have a better correspondence with the experiment $\mathrm{CD}$, the calculation process uses the PCM implicit solvent model.

\section{Reference}

Tian

Lu, $\quad$ molclus

program, Version

1.9. http://www.keinsci.com/research/molclus.html (accessed 2019-Nov-5).

(2) Grimme, S.; Bannwarth, C.; Shushkov, P. J. Chem. Theory Comput. 2017, 13, 1989-2009.
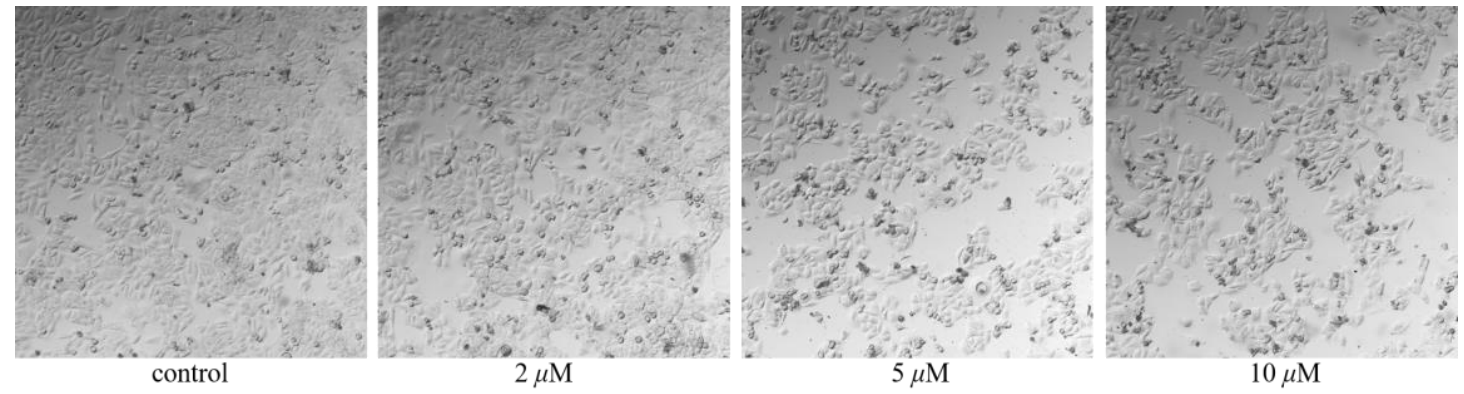

Figure S1. The effect of $\mathbf{1}$ in different concentrations on morphology of BEL-7402 cells under microscope. The experimental results showed that compared with the control group, the cell density of the dosing treatment group was significantly reduced, 
the cell viability was weakened, the dead cells were round, the cell adhesion was significantly weakened, the connections between the cells were weakened, and the cell debris was significantly increased, which indicated BEL-7402 cells were killed by compound 1. So, from the figure, it can be seen that the addition of $\mathbf{1}$ to the 96 -well plates will lead to the death of tumor cells, which is concentration-dependent.
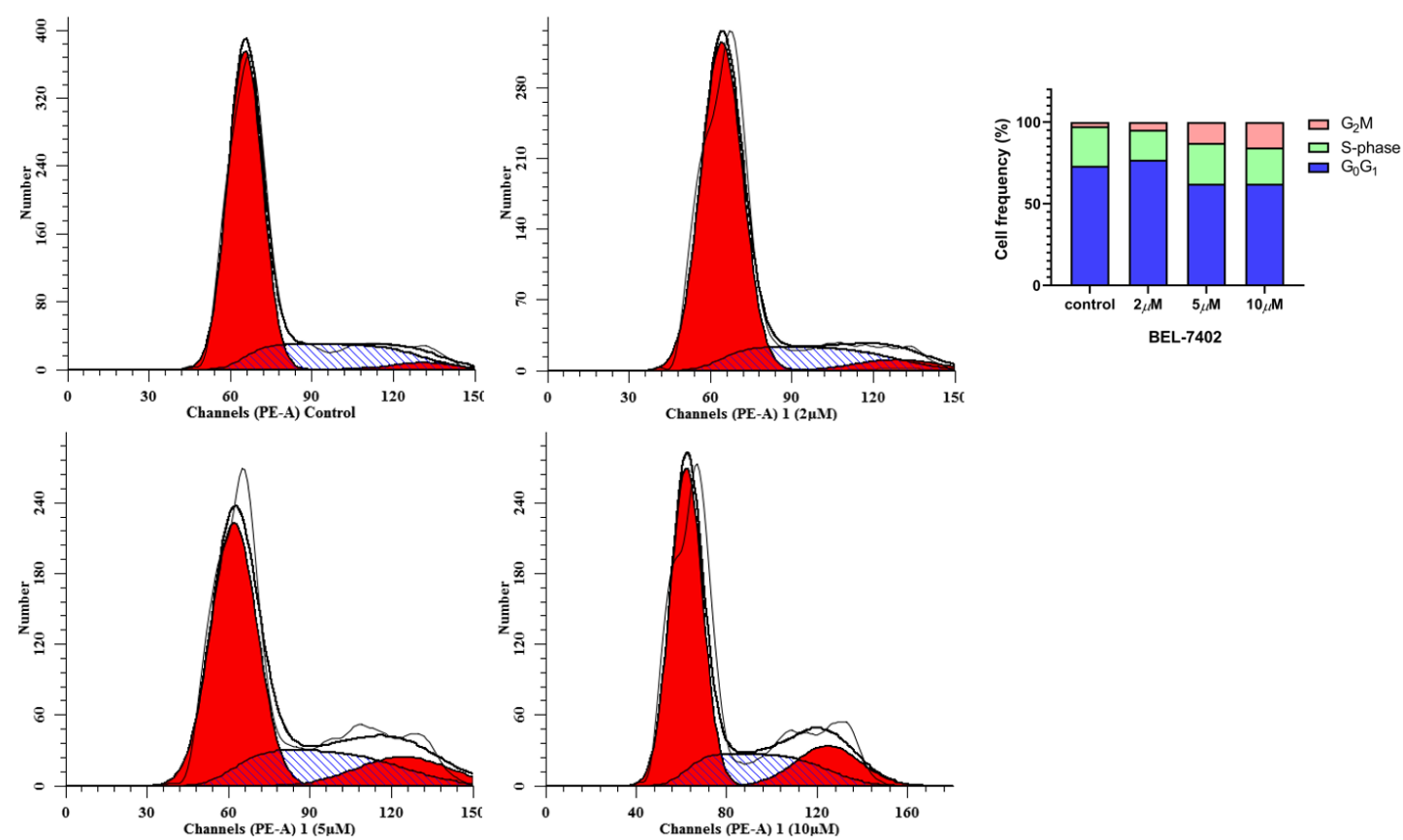

Figure S2. (A) The effect of $\mathbf{1}$ on cell cycle. (B) Compound $\mathbf{1}$ dose dependently induced G2/M phase arrest of BEL-7402 cells.

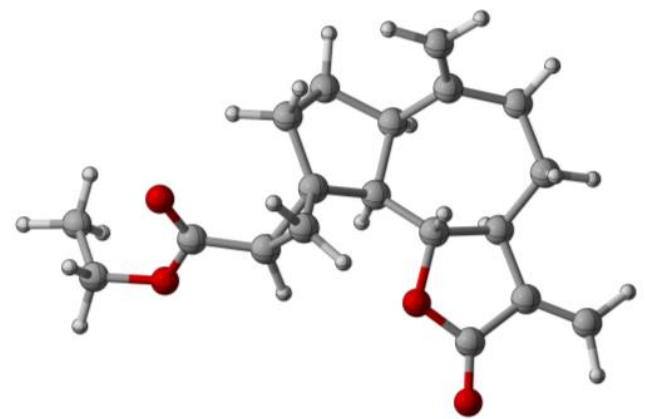

Figure S3. PBE1PBE/TZVP optimized lowest energy 3D conformer of $\mathbf{1}$. 
Table S1. The Cartesian coordinates for the lowest-energy conformer of $\mathbf{1}$ in the ECD calculations.

\begin{tabular}{cccccccc}
\hline Atom & $\mathrm{X}$ & $\mathrm{Y}$ & $\mathrm{Z}$ & $\mathrm{Atom}$ & $\mathrm{X}$ & $\mathrm{Y}$ & $\mathrm{Z}$ \\
\hline $\mathrm{C}$ & -0.64719 & 2.5308 & -0.7973 & $\mathrm{H}$ & 1.0777 & 1.8007 & -1.87441 \\
$\mathrm{C}$ & -1.38138 & 1.94108 & 0.40487 & $\mathrm{H}$ & -0.56165 & 3.61923 & -0.75833 \\
$\mathrm{C}$ & -0.86815 & 0.50912 & 0.50278 & $\mathrm{H}$ & -1.1894 & 2.27798 & -1.71471 \\
$\mathrm{C}$ & 0.37807 & 0.38508 & -0.39692 & $\mathrm{H}$ & -2.4662 & 1.99482 & 0.30612 \\
$\mathrm{C}$ & 0.7211 & 1.83953 & -0.83929 & $\mathrm{H}$ & -1.12411 & 2.4765 & 1.32368 \\
$\mathrm{C}$ & 1.54249 & -0.35794 & 0.2191 & $\mathrm{H}$ & 1.72283 & $-3.00 \mathrm{E}-05$ & 1.2393 \\
$\mathrm{C}$ & 2.85835 & -0.31947 & -0.57286 & $\mathrm{H}$ & 2.61988 & -0.22411 & -1.64238 \\
$\mathrm{C}$ & 3.77038 & 0.82303 & -0.14973 & $\mathrm{H}$ & 4.74926 & 0.69874 & -0.62369 \\
$\mathrm{C}$ & 3.23498 & 2.21165 & -0.50976 & $\mathrm{H}$ & 3.9325 & 0.75772 & 0.93257 \\
$\mathrm{C}$ & 1.82837 & 2.51041 & -0.04461 & $\mathrm{H}$ & 3.91702 & 2.9591 & -0.09483 \\
$\mathrm{C}$ & 1.61865 & 3.36959 & 0.95351 & $\mathrm{H}$ & 3.26734 & 2.32382 & -1.60166 \\
$\mathrm{O}$ & 1.1921 & -1.76182 & 0.32993 & $\mathrm{H}$ & 0.62616 & 3.6389 & 1.2972 \\
$\mathrm{C}$ & 2.2608 & -2.5503 & 0.10391 & $\mathrm{H}$ & 2.4536 & 3.84687 & 1.45846 \\
$\mathrm{C}$ & 3.39441 & -1.70066 & -0.3387 & $\mathrm{H}$ & 5.46489 & -1.56722 & -0.76152 \\
$\mathrm{C}$ & 4.62673 & -2.18321 & -0.45453 & $\mathrm{H}$ & 4.81751 & -3.22892 & -0.23559 \\
$\mathrm{O}$ & 2.21878 & -3.74244 & 0.2545 & $\mathrm{H}$ & -1.46472 & -1.57257 & 0.01851 \\
$\mathrm{H}$ & 0.09523 & -0.16674 & -1.30156 & $\mathrm{H}$ & -0.26377 & -0.93925 & 2.10074 \\
$\mathrm{C}$ & -1.81623 & -0.69262 & 0.54385 & $\mathrm{H}$ & -1.62176 & 0.21655 & 2.54305 \\
$\mathrm{C}$ & -1.04072 & -0.26459 & 1.76294 & $\mathrm{H}$ & -5.58551 & -2.25383 & -0.81255 \\
$\mathrm{C}$ & -3.27791 & -0.49963 & 0.51987 & $\mathrm{H}$ & -5.72028 & -1.0561 & 0.49563 \\
$\mathrm{O}$ & -3.85528 & -1.33657 & -0.35358 & $\mathrm{H}$ & -6.77293 & -0.21852 & -1.62851 \\
$\mathrm{O}$ & -3.8955 & 0.29891 & 1.1897 & $\mathrm{H}$ & -5.40101 & 0.79286 & -1.1474 \\
$\mathrm{C}$ & -5.28705 & -1.25722 & -0.48579 & $\mathrm{H}$ & -5.21832 & -0.39169 & -2.46014 \\
$\mathrm{C}$ & -5.68821 & -0.20292 & -1.49173 & & & & \\
\hline & & & & & & &
\end{tabular}

Table S2. The single point energy for the lowest-energy conformer of $\mathbf{1}$ in the ECD calculations.

\begin{tabular}{cc}
\hline Conf. & Single Point Energy (Hartree, a.u.) \\
\hline 1 & -1038.420328 \\
\hline
\end{tabular}

Table S3. The imaginary frequencies for the lowest-energy conformer of $\mathbf{1}$.

\begin{tabular}{cccccc}
\hline No. & Freq. $(\mathrm{Hz})$ & IR & No. & Freq. $(\mathrm{Hz})$ & IR \\
\hline 1 & -500 & 0 & 251 & 1500 & -44.7413 \\
2 & -492 & 0 & 252 & 1508 & -6.16411 \\
3 & -484 & 0 & 253 & 1516 & -1.68536 \\
4 & -476 & 0 & 254 & 1524 & -0.70128 \\
5 & -468 & 0 & 255 & 1532 & -0.36436 \\
6 & -460 & 0 & 256 & 1540 & -0.21573 \\
& & & S5
\end{tabular}




\begin{tabular}{|c|c|c|c|c|c|}
\hline 7 & -452 & 0 & 257 & 1548 & -0.13983 \\
\hline 8 & -444 & 0 & 258 & 1556 & -0.09609 \\
\hline 9 & -436 & 0 & 259 & 1564 & -0.06845 \\
\hline 10 & -428 & 0 & 260 & 1572 & -0.04964 \\
\hline 11 & -420 & 0 & 261 & 1580 & -0.0377 \\
\hline 12 & -412 & 0 & 262 & 1588 & -0.02698 \\
\hline 13 & -404 & 0 & 263 & 1596 & -0.01721 \\
\hline 14 & -396 & 0 & 264 & 1604 & -0.01245 \\
\hline 15 & -388 & 0 & 265 & 1612 & -0.00873 \\
\hline 16 & -380 & 0 & 266 & 1620 & -0.00542 \\
\hline 17 & -372 & 0 & 267 & 1628 & 0.009003 \\
\hline 18 & -364 & 0 & 268 & 1636 & 0.015134 \\
\hline 19 & -356 & 0 & 269 & 1644 & 0.022025 \\
\hline 20 & -348 & 0 & 270 & 1652 & 0.031443 \\
\hline 21 & -340 & 0 & 271 & 1660 & 0.045128 \\
\hline 22 & -332 & 0 & 272 & 1668 & 0.061507 \\
\hline 23 & -324 & 0 & 273 & 1676 & 0.088893 \\
\hline 24 & -316 & 0 & 274 & 1684 & 0.15861 \\
\hline 25 & -308 & 0 & 275 & 1692 & 0.277907 \\
\hline 26 & -300 & 0 & 276 & 1700 & 0.632778 \\
\hline 27 & -292 & 0 & 277 & 1708 & 2.418154 \\
\hline 28 & -284 & 0 & 278 & 1716 & 18.399 \\
\hline 29 & -276 & 0 & 279 & 1724 & -10.1646 \\
\hline 30 & -268 & 0 & 280 & 1732 & -0.11184 \\
\hline 31 & -260 & 0 & 281 & 1740 & 6.523407 \\
\hline 32 & -252 & 0 & 282 & 1748 & -13.8742 \\
\hline 33 & -244 & 0 & 283 & 1756 & -0.42868 \\
\hline 34 & -236 & 0 & 284 & 1764 & 4.709045 \\
\hline 35 & -228 & 0 & 285 & 1772 & 21.02983 \\
\hline 36 & -220 & 0 & 286 & 1780 & 176.343 \\
\hline 37 & -212 & 0 & 287 & 1788 & -155.662 \\
\hline 38 & -204 & 0 & 288 & 1796 & -18.2316 \\
\hline 39 & -196 & 0 & 289 & 1804 & -3.50664 \\
\hline 40 & -188 & 0 & 290 & 1812 & 0.694102 \\
\hline 41 & -180 & 0 & 291 & 1820 & 5.285996 \\
\hline 42 & -172 & 0 & 292 & 1828 & 21.07055 \\
\hline 43 & -164 & 0 & 293 & 1836 & 150.0804 \\
\hline 44 & -156 & 0 & 294 & 1844 & -349.949 \\
\hline 45 & -148 & 0 & 295 & 1852 & -56.4187 \\
\hline 46 & -140 & $4.8 \mathrm{E}-05$ & 296 & 1860 & -11.6767 \\
\hline 47 & -132 & $5.61 \mathrm{E}-05$ & 297 & 1868 & -4.07384 \\
\hline 48 & -124 & $6.62 \mathrm{E}-05$ & 298 & 1876 & -1.87052 \\
\hline 49 & -116 & 0.000128 & 299 & 1884 & -1.01159 \\
\hline 50 & -108 & 0.000178 & 300 & 1892 & -0.61009 \\
\hline
\end{tabular}




\begin{tabular}{|c|c|c|c|c|c|}
\hline 51 & -100 & 0.000289 & 301 & 1900 & -0.39725 \\
\hline 52 & -92 & 0.00039 & 302 & 1908 & -0.27305 \\
\hline 53 & -84 & 0.000504 & 303 & 1916 & -0.19646 \\
\hline 54 & -76 & 0.000618 & 304 & 1924 & -0.14633 \\
\hline 55 & -68 & 0.000772 & 305 & 1932 & -0.11208 \\
\hline 56 & -60 & 0.000983 & 306 & 1940 & -0.08786 \\
\hline 57 & -52 & 0.001409 & 307 & 1948 & -0.06063 \\
\hline 58 & -44 & 0.00188 & 308 & 1956 & -0.04874 \\
\hline 59 & -36 & 0.002603 & 309 & 1964 & -0.03976 \\
\hline 60 & -28 & 0.003817 & 310 & 1972 & -0.03286 \\
\hline 61 & -20 & 0.00602 & 311 & 1980 & -0.02747 \\
\hline 62 & -12 & 0.0107 & 312 & 1988 & -0.0232 \\
\hline 63 & -4 & 0.023306 & 313 & 1996 & -0.01977 \\
\hline 64 & 4 & 0.074983 & 314 & 2004 & 0 \\
\hline 65 & 12 & 0.517947 & 315 & 2012 & 0 \\
\hline 66 & 20 & -0.89921 & 316 & 2020 & 0 \\
\hline 67 & 28 & 0.009899 & 317 & 2028 & 0 \\
\hline 68 & 36 & 0.898101 & 318 & 2036 & 0 \\
\hline 69 & 44 & -0.01735 & 319 & 2044 & 0 \\
\hline 70 & 52 & 0.695479 & 320 & 2052 & 0 \\
\hline 71 & 60 & -0.9275 & 321 & 2060 & 0 \\
\hline 72 & 68 & -0.84466 & 322 & 2068 & 0 \\
\hline 73 & 76 & -0.28182 & 323 & 2076 & 0 \\
\hline 74 & 84 & -0.12155 & 324 & 2084 & 0 \\
\hline 75 & 92 & 0.218625 & 325 & 2092 & 0 \\
\hline 76 & 100 & 2.140238 & 326 & 2100 & 0 \\
\hline 77 & 108 & -1.47974 & 327 & 2108 & 0 \\
\hline 78 & 116 & -0.19536 & 328 & 2116 & 0 \\
\hline 79 & 124 & 0.181678 & 329 & 2124 & 0 \\
\hline 80 & 132 & -0.21437 & 330 & 2132 & 0 \\
\hline 81 & 140 & 0.008983 & 331 & 2140 & 0 \\
\hline 82 & 148 & 0.174455 & 332 & 2148 & 0 \\
\hline 83 & 156 & 1.456358 & 333 & 2156 & 0 \\
\hline 84 & 164 & -0.76549 & 334 & 2164 & 0 \\
\hline 85 & 172 & 0.662104 & 335 & 2172 & 0 \\
\hline 86 & 180 & -0.42582 & 336 & 2180 & 0 \\
\hline 87 & 188 & 0.120876 & 337 & 2188 & 0 \\
\hline 88 & 196 & 1.504421 & 338 & 2196 & 0 \\
\hline 89 & 204 & -0.96322 & 339 & 2204 & 0 \\
\hline 90 & 212 & -0.05431 & 340 & 2212 & 0 \\
\hline 91 & 220 & 0.316732 & 341 & 2220 & 0 \\
\hline 92 & 228 & 1.207297 & 342 & 2228 & 0 \\
\hline 93 & 236 & -0.89981 & 343 & 2236 & 0 \\
\hline 94 & 244 & -0.27906 & 344 & 2244 & 0 \\
\hline
\end{tabular}




\begin{tabular}{|c|c|c|c|c|c|}
\hline 95 & 252 & -0.07338 & 345 & 2252 & 0 \\
\hline 96 & 260 & 0.083611 & 346 & 2260 & 0 \\
\hline 97 & 268 & 0.809936 & 347 & 2268 & 0 \\
\hline 98 & 276 & -0.86175 & 348 & 2276 & 0 \\
\hline 99 & 284 & 0.078301 & 349 & 2284 & 0 \\
\hline 100 & 292 & 1.20857 & 350 & 2292 & 0 \\
\hline 101 & 300 & -1.18406 & 351 & 2300 & 0 \\
\hline 102 & 308 & 0.332238 & 352 & 2308 & 0 \\
\hline 103 & 316 & -0.16356 & 353 & 2316 & 0 \\
\hline 104 & 324 & 1.574786 & 354 & 2324 & 0 \\
\hline 105 & 332 & -1.60036 & 355 & 2332 & 0 \\
\hline 106 & 340 & 0.078184 & 356 & 2340 & 0 \\
\hline 107 & 348 & 1.628407 & 357 & 2348 & 0 \\
\hline 108 & 356 & 5.650133 & 358 & 2356 & 0 \\
\hline 109 & 364 & -1.96264 & 359 & 2364 & 0 \\
\hline 110 & 372 & -0.12364 & 360 & 2372 & 0 \\
\hline 111 & 380 & 4.431144 & 361 & 2380 & 0 \\
\hline 112 & 388 & -1.93602 & 362 & 2388 & 0 \\
\hline 113 & 396 & 0.486316 & 363 & 2396 & 0 \\
\hline 114 & 404 & -1.68555 & 364 & 2404 & 0 \\
\hline 115 & 412 & 0.054182 & 365 & 2412 & 0 \\
\hline 116 & 420 & -0.44074 & 366 & 2420 & 0 \\
\hline 117 & 428 & -0.05301 & 367 & 2428 & 0 \\
\hline 118 & 436 & 0.030927 & 368 & 2436 & 0 \\
\hline 119 & 444 & 0.223176 & 369 & 2444 & 0 \\
\hline 120 & 452 & 1.477024 & 370 & 2452 & 0 \\
\hline 121 & 460 & -0.48918 & 371 & 2460 & 0 \\
\hline 122 & 468 & 0.603431 & 372 & 2468 & 0 \\
\hline 123 & 476 & 2.797066 & 373 & 2476 & 0 \\
\hline 124 & 484 & -0.56185 & 374 & 2484 & 0 \\
\hline 125 & 492 & -0.80994 & 375 & 2492 & 0 \\
\hline 126 & 500 & 0.243962 & 376 & 2500 & 0 \\
\hline 127 & 508 & 0.905861 & 377 & 2508 & 0 \\
\hline 128 & 516 & 5.96173 & 378 & 2516 & 0 \\
\hline 129 & 524 & -4.01293 & 379 & 2524 & 0 \\
\hline 130 & 532 & -0.52197 & 380 & 2532 & 0 \\
\hline 131 & 540 & -0.13605 & 381 & 2540 & 0 \\
\hline 132 & 548 & -0.04291 & 382 & 2548 & 0 \\
\hline 133 & 556 & 0.004327 & 383 & 2556 & 0 \\
\hline 134 & 564 & 0.07988 & 384 & 2564 & 0 \\
\hline 135 & 572 & 0.471758 & 385 & 2572 & 0 \\
\hline 136 & 580 & 1.987388 & 386 & 2580 & 0 \\
\hline 137 & 588 & -0.88604 & 387 & 2588 & 0 \\
\hline 138 & 596 & -0.11718 & 388 & 2596 & 0 \\
\hline
\end{tabular}




\begin{tabular}{|c|c|c|c|c|c|}
\hline 139 & 604 & $9.82 \mathrm{E}-05$ & 389 & 2604 & 0 \\
\hline 140 & 612 & 0.078906 & 390 & 2612 & 0 \\
\hline 141 & 620 & 0.313483 & 391 & 2620 & 0 \\
\hline 142 & 628 & 2.258621 & 392 & 2628 & 0 \\
\hline 143 & 636 & -4.37525 & 393 & 2636 & 0 \\
\hline 144 & 644 & -0.38494 & 394 & 2644 & 0 \\
\hline 145 & 652 & 0.620622 & 395 & 2652 & 0 \\
\hline 146 & 660 & 5.7074 & 396 & 2660 & 0 \\
\hline 147 & 668 & -8.75679 & 397 & 2668 & 0 \\
\hline 148 & 676 & -0.70381 & 398 & 2676 & 0 \\
\hline 149 & 684 & 1.385313 & 399 & 2684 & 0 \\
\hline 150 & 692 & 5.775224 & 400 & 2692 & 0 \\
\hline 151 & 700 & -2.68214 & 401 & 2700 & 0 \\
\hline 152 & 708 & 0.25546 & 402 & 2708 & 0 \\
\hline 153 & 716 & 5.06149 & 403 & 2716 & 0 \\
\hline 154 & 724 & -2.71514 & 404 & 2724 & 0 \\
\hline 155 & 732 & -0.2158 & 405 & 2732 & 0 \\
\hline 156 & 740 & 0.92209 & 406 & 2740 & 0 \\
\hline 157 & 748 & -0.9478 & 407 & 2748 & 0 \\
\hline 158 & 756 & -2.03 & 408 & 2756 & 0 \\
\hline 159 & 764 & -0.08908 & 409 & 2764 & 0 \\
\hline 160 & 772 & 1.656037 & 410 & 2772 & 0 \\
\hline 161 & 780 & 5.04483 & 411 & 2780 & 0 \\
\hline 162 & 788 & -2.64007 & 412 & 2788 & 0 \\
\hline 163 & 796 & -0.24319 & 413 & 2796 & 0 \\
\hline 164 & 804 & 0.833541 & 414 & 2804 & 0 \\
\hline 165 & 812 & 1.443478 & 415 & 2812 & 0 \\
\hline 166 & 820 & -1.63851 & 416 & 2820 & 0 \\
\hline 167 & 828 & 0.996924 & 417 & 2828 & 0 \\
\hline 168 & 836 & 7.473063 & 418 & 2836 & 0 \\
\hline 169 & 844 & 2.514498 & 419 & 2844 & 0 \\
\hline 170 & 852 & 3.09186 & 420 & 2852 & 0 \\
\hline 171 & 860 & -5.03475 & 421 & 2860 & 0.000239 \\
\hline 172 & 868 & 2.958024 & 422 & 2868 & 0.000279 \\
\hline 173 & 876 & 2.338801 & 423 & 2876 & 0.001212 \\
\hline 174 & 884 & -10.4494 & 424 & 2884 & 0.001425 \\
\hline 175 & 892 & -1.50717 & 425 & 2892 & 0.002773 \\
\hline 176 & 900 & -0.16022 & 426 & 2900 & 0.004592 \\
\hline 177 & 908 & 1.423465 & 427 & 2908 & 0.006039 \\
\hline 178 & 916 & 1.667917 & 428 & 2916 & 0.011492 \\
\hline 179 & 924 & 16.22004 & 429 & 2924 & 0.013801 \\
\hline 180 & 932 & -28.1406 & 430 & 2932 & 0.016792 \\
\hline 181 & 940 & -10.999 & 431 & 2940 & 0.023256 \\
\hline 182 & 948 & -7.39632 & 432 & 2948 & 0.03064 \\
\hline
\end{tabular}




\begin{tabular}{|c|c|c|c|c|c|}
\hline 183 & 956 & -1.8561 & 433 & 2956 & 0.038915 \\
\hline 184 & 964 & 0.912856 & 434 & 2964 & 0.051999 \\
\hline 185 & 972 & 7.853629 & 435 & 2972 & 0.069967 \\
\hline 186 & 980 & 11.53589 & 436 & 2980 & 0.099932 \\
\hline 187 & 988 & -7.74864 & 437 & 2988 & 0.151782 \\
\hline 188 & 996 & 0.171546 & 438 & 2996 & 0.258734 \\
\hline 189 & 1004 & -4.78152 & 439 & 3004 & 0.560327 \\
\hline 190 & 1012 & 10.66445 & 440 & 3012 & 2.205662 \\
\hline 191 & 1020 & -2.51458 & 441 & 3020 & -1.03462 \\
\hline 192 & 1028 & 8.038727 & 442 & 3028 & 13.14511 \\
\hline 193 & 1036 & 24.83333 & 443 & 3036 & -10.3656 \\
\hline 194 & 1044 & 58.66407 & 444 & 3044 & 14.56662 \\
\hline 195 & 1052 & -36.1696 & 445 & 3052 & 3.070471 \\
\hline 196 & 1060 & -1.27249 & 446 & 3060 & -2.84628 \\
\hline 197 & 1068 & 23.8253 & 447 & 3068 & 53.5314 \\
\hline 198 & 1076 & -15.919 & 448 & 3076 & -50.6295 \\
\hline 199 & 1084 & -0.08238 & 449 & 3084 & -2.35742 \\
\hline 200 & 1092 & -6.28113 & 450 & 3092 & 32.91811 \\
\hline 201 & 1100 & -0.15873 & 451 & 3100 & -17.5296 \\
\hline 202 & 1108 & 17.29196 & 452 & 3108 & -25.4529 \\
\hline 203 & 1116 & -0.70346 & 453 & 3116 & 19.35703 \\
\hline 204 & 1124 & -11.4229 & 454 & 3124 & -11.7629 \\
\hline 205 & 1132 & -17.5141 & 455 & 3132 & 18.9606 \\
\hline 206 & 1140 & 2.147742 & 456 & 3140 & -10.5597 \\
\hline 207 & 1148 & 33.19809 & 457 & 3148 & 2.640772 \\
\hline 208 & 1156 & -13.7975 & 458 & 3156 & 3.798597 \\
\hline 209 & 1164 & 10.72933 & 459 & 3164 & -25.247 \\
\hline 210 & 1172 & 88.45183 & 460 & 3172 & -4.36247 \\
\hline 211 & 1180 & 32.47034 & 461 & 3180 & -1.06847 \\
\hline 212 & 1188 & 8.619756 & 462 & 3188 & -0.39139 \\
\hline 213 & 1196 & 66.76676 & 463 & 3196 & 0.035506 \\
\hline 214 & 1204 & 4.55836 & 464 & 3204 & 0.696354 \\
\hline 215 & 1212 & -73.8217 & 465 & 3212 & -0.43692 \\
\hline 216 & 1220 & -39.3073 & 466 & 3220 & 0.042321 \\
\hline 217 & 1228 & -2.44318 & 467 & 3228 & 0.53087 \\
\hline 218 & 1236 & 25.86499 & 468 & 3236 & 4.03569 \\
\hline 219 & 1244 & -44.3472 & 469 & 3244 & -8.16904 \\
\hline 220 & 1252 & -1.58955 & 470 & 3252 & -2.02454 \\
\hline 221 & 1260 & 28.15483 & 471 & 3260 & -0.92397 \\
\hline 222 & 1268 & -6.06851 & 472 & 3268 & -0.30412 \\
\hline 223 & 1276 & 47.57325 & 473 & 3276 & -0.09771 \\
\hline 224 & 1284 & 67.73671 & 474 & 3284 & -0.04731 \\
\hline 225 & 1292 & -36.4082 & 475 & 3292 & -0.02809 \\
\hline 226 & 1300 & 3.962054 & 476 & 3300 & -0.01756 \\
\hline
\end{tabular}




\begin{tabular}{cccccc}
227 & 1308 & -0.18383 & 477 & 3308 & -0.0117 \\
228 & 1316 & 45.14082 & 478 & 3316 & -0.00788 \\
229 & 1324 & -29.1151 & 479 & 3324 & -0.00486 \\
230 & 1332 & 26.53887 & 480 & 3332 & -0.00353 \\
231 & 1340 & -40.0399 & 481 & 3340 & -0.00272 \\
232 & 1348 & -4.57185 & 482 & 3348 & -0.00214 \\
233 & 1356 & 45.57893 & 483 & 3356 & -0.00172 \\
234 & 1364 & -16.4666 & 484 & 3364 & -0.0014 \\
235 & 1372 & 5.344572 & 485 & 3372 & -0.0011 \\
236 & 1380 & -15.0335 & 486 & 3380 & -0.00091 \\
237 & 1388 & 7.16593 & 487 & 3388 & -0.00077 \\
238 & 1396 & -4.45998 & 488 & 3396 & -0.00065 \\
239 & 1404 & 15.87424 & 489 & 3404 & -0.00011 \\
240 & 1412 & -21.2771 & 490 & 3412 & $-4.6 \mathrm{E}-05$ \\
241 & 1420 & 19.43268 & 491 & 3420 & 0 \\
242 & 1428 & 26.69263 & 492 & 3428 & 0 \\
243 & 1436 & -66.3326 & 493 & 3436 & 0 \\
244 & 1444 & -12.116 & 494 & 3444 & 0 \\
245 & 1452 & -1.83485 & 495 & 3452 & 0 \\
246 & 1460 & 1.855981 & 496 & 3460 & 0 \\
247 & 1468 & 17.19168 & 497 & 3468 & 0 \\
248 & 1476 & 5.882157 & 498 & 3476 & 0 \\
249 & 1484 & 20.84064 & 499 & 3484 & 0 \\
250 & 1492 & 34.0182 & 500 & 3492 & 0 \\
\hline & & & & & 0 \\
\hline
\end{tabular}

Table S4. Crystal data and structure refinement for $\mathbf{1 .}$

Empirical formula $\quad \mathrm{C}_{19} \mathrm{H}_{24} \mathrm{O}_{4}$

Formula weight $\quad 316.38$

Temperature 293(2) K

radiation type $\quad \mathrm{Cu} \mathrm{K} \backslash \alpha$ '

Bond precision $\quad \mathrm{C}-\mathrm{C}=0.0036 \AA$

Wavelength $1.54184 \AA$

Crystal system Orthorhombic

Space group $\quad$ P 212121

Unit cell dimensions a $=8.79521(11) \AA \quad=90^{\circ}$

$$
\begin{array}{ll}
\mathrm{b}=9.11180(9) \AA & =90^{\circ} \\
\mathrm{c}=21.6544(3) \AA & =90^{\circ}
\end{array}
$$


Volume 1735.39(4) $\AA^{3}$

$\mathrm{Z} 4$

Density diffrn $\quad 1.211 \mathrm{Mg} / \mathrm{m}^{3}$

Absorption coefficient $0.678 \mathrm{~mm}^{-1}$

$\mathrm{F}(000) 680$

Crystal size 0.14 x $0.13 \times 0.06 \mathrm{~mm}^{3}$

Theta range for data collection $4.8470^{\circ}$ to $69.8460^{\circ}$

Index ranges $\quad-10<=\mathrm{h}<=10,-10<=\mathrm{k}<=10,-25<=\mathrm{l}<=25$

Tmin, Tmax $\quad 0.909,0.960$

Reflections collected 3060

Independent reflections 2956 [R(int) $=0.0361]$

Completeness to theta $=66.97^{\circ} 99.89 \%$

Data / restraints / parameters 3060 / 12 / 227

Goodness-of-fit on F2 1.052

$\mathrm{R}($ reflections $)=0.0361(2956), \mathrm{wR} 2($ reflections $)=0.1044(3060)$

Absolute structure Flack 0.09(7)

Extinction coefficient $0.0067(8)$

Largest diff. peak and hole $\quad 0.158$ and -0.120 e. $\AA^{-3}$

${ }^{\mathrm{a} C}$ Colorless crystals of $\mathbf{1}$ was obtained in $\mathrm{CH}_{2} \mathrm{Cl}_{2}$. 


\section{Mass Spectrum SmartFormula Report}

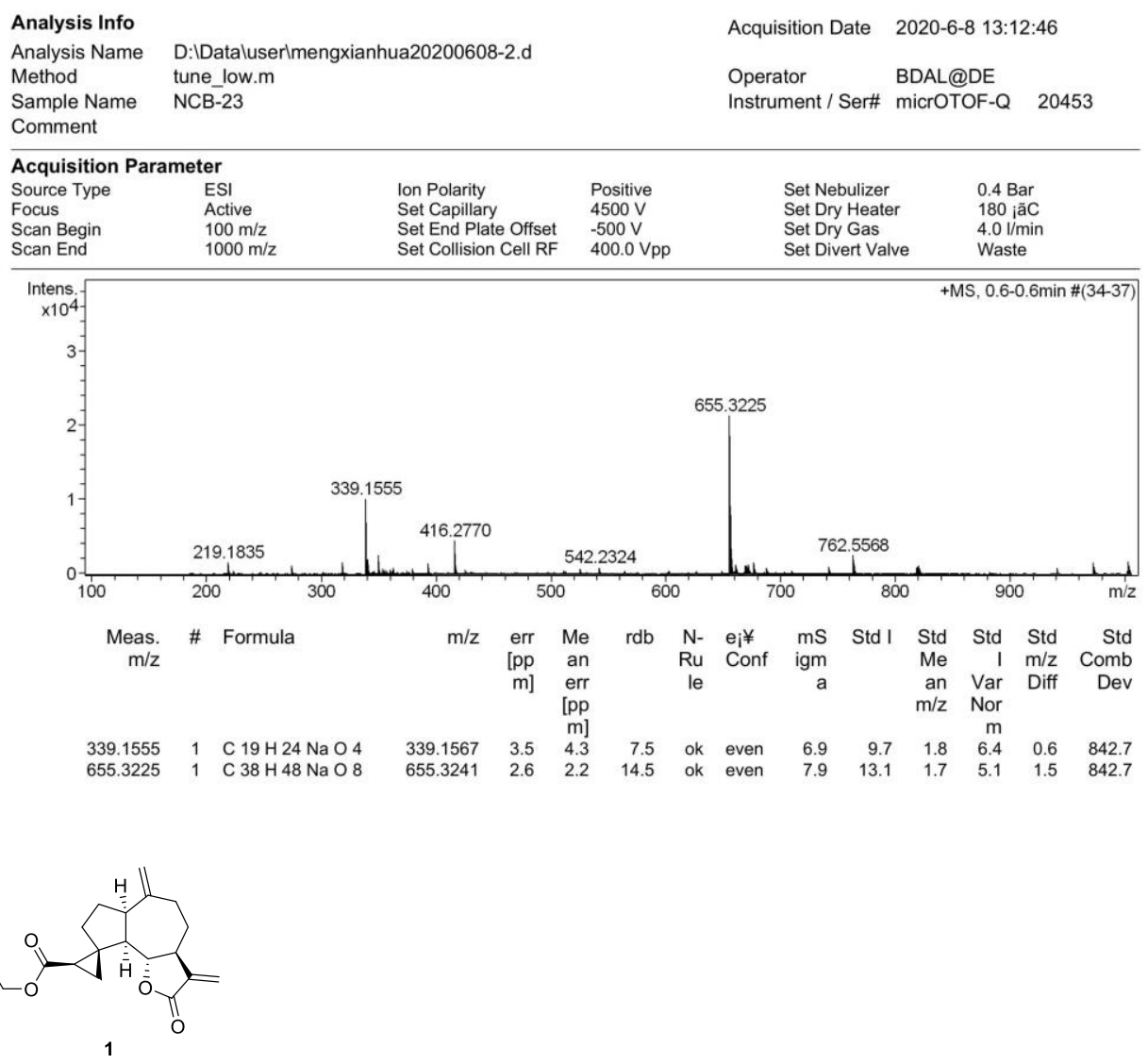

Figure S4. HRESIMS Spectrum of 1 


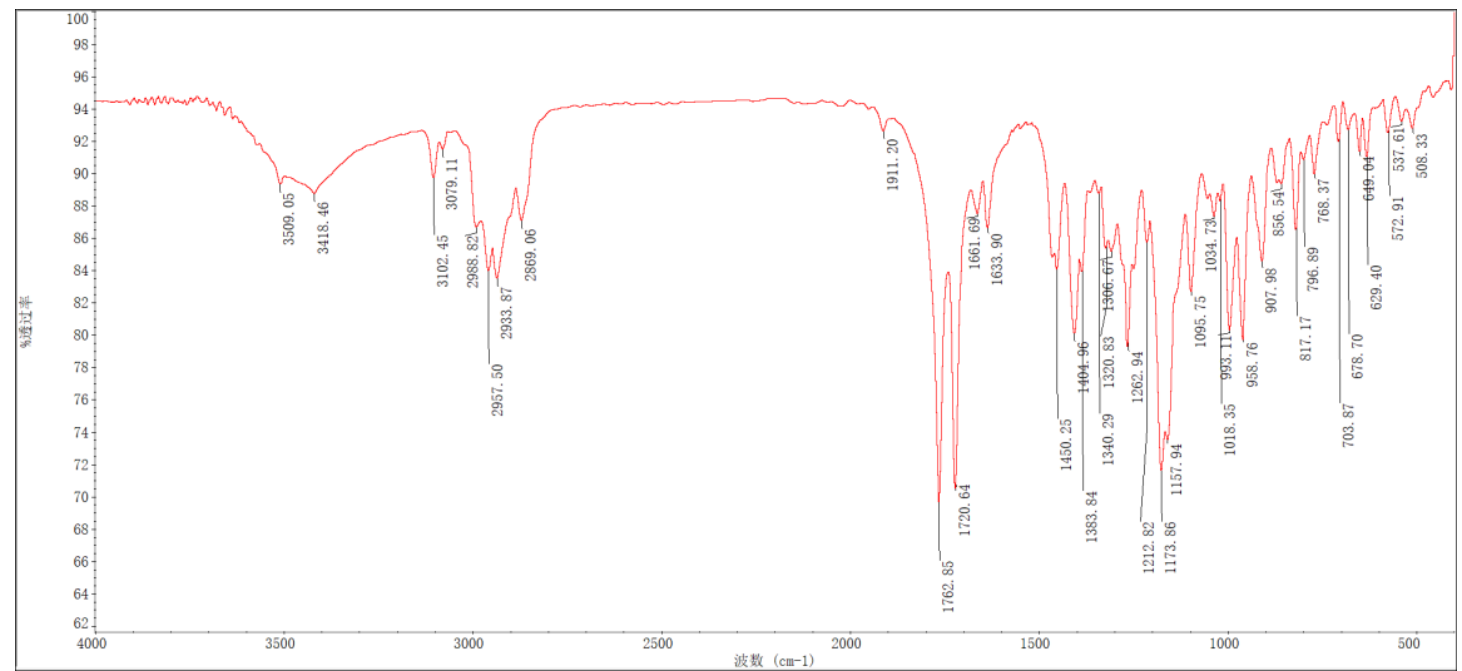

Figure S5. IR Spectrum of 1
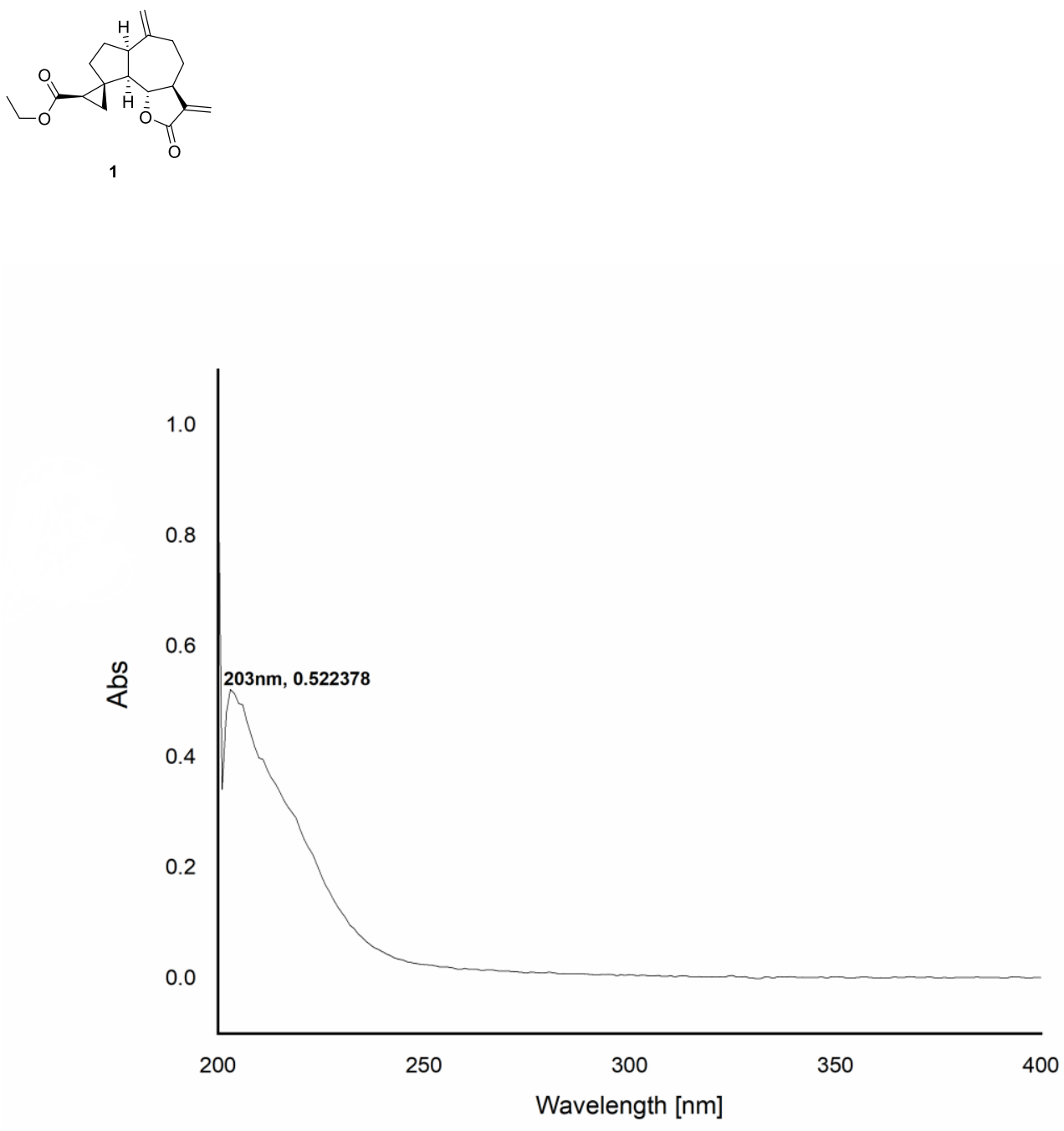

Figure S6. UV Spectrum of $1(\mathrm{MeOH})$ 

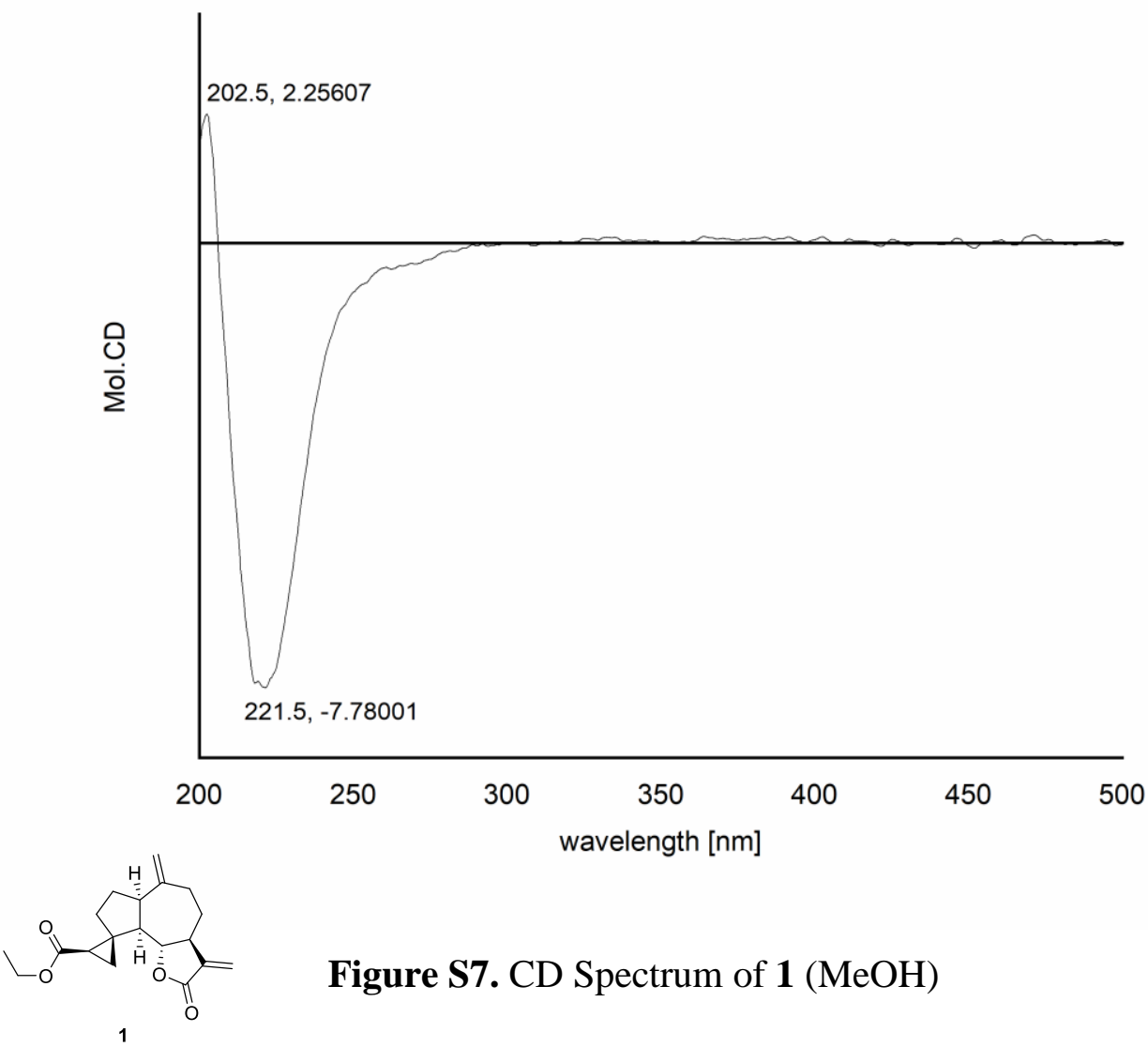

Figure S7. CD Spectrum of $1(\mathrm{MeOH})$

PROTON CDCl3 \{E: \data\} ROOT 8

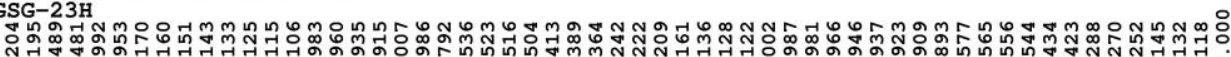

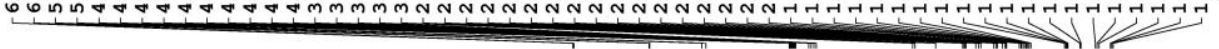

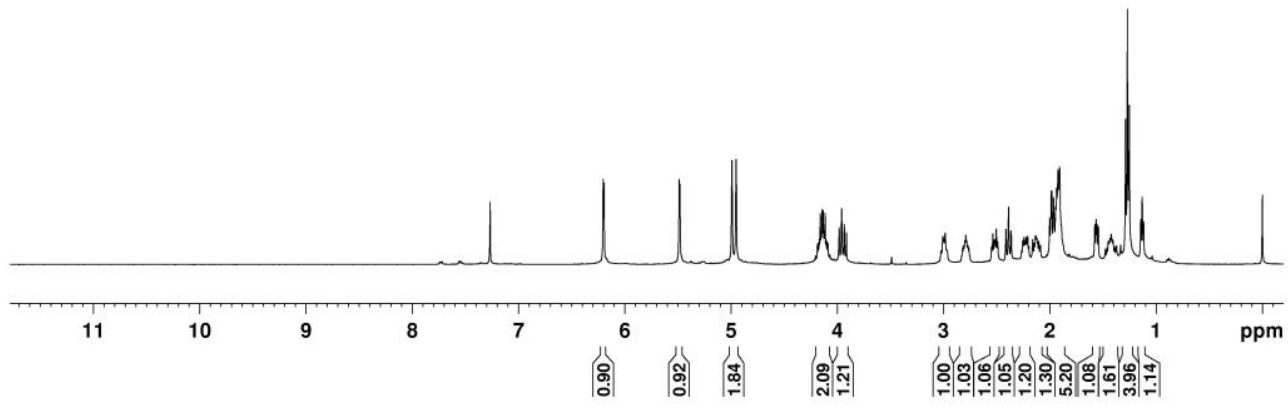

Figure S8. ${ }^{1} \mathrm{H}$ NMR Spectrum of $1\left(400 \mathrm{MHz}, \mathrm{CDCl}_{3}\right)$ 
C13CPD CDCl3 \{E: \data\} ROOT 3 GSG-23C_20200310

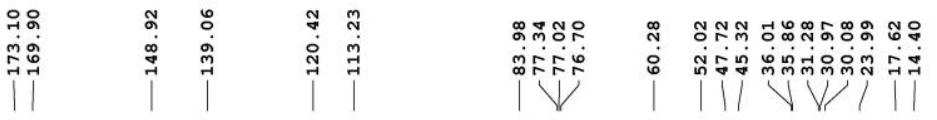
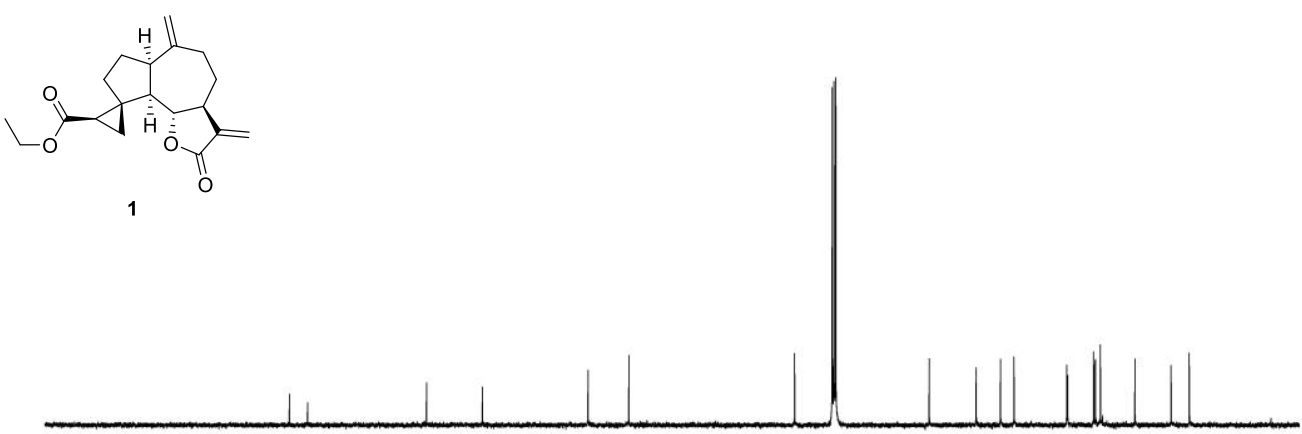

$\begin{array}{llllllllllllllllllllll}210 & 200 & 190 & 180 & 170 & 160 & 150 & 140 & 130 & 120 & 110 & 100 & 90 & 80 & 70 & 60 & 50 & 40 & 30 & 20 & 10 & \mathrm{ppm}\end{array}$

Figure S9. ${ }^{13} \mathrm{C}$ NMR Spectrum of $1\left(100 \mathrm{MHz}, \mathrm{CDCl}_{3}\right)$

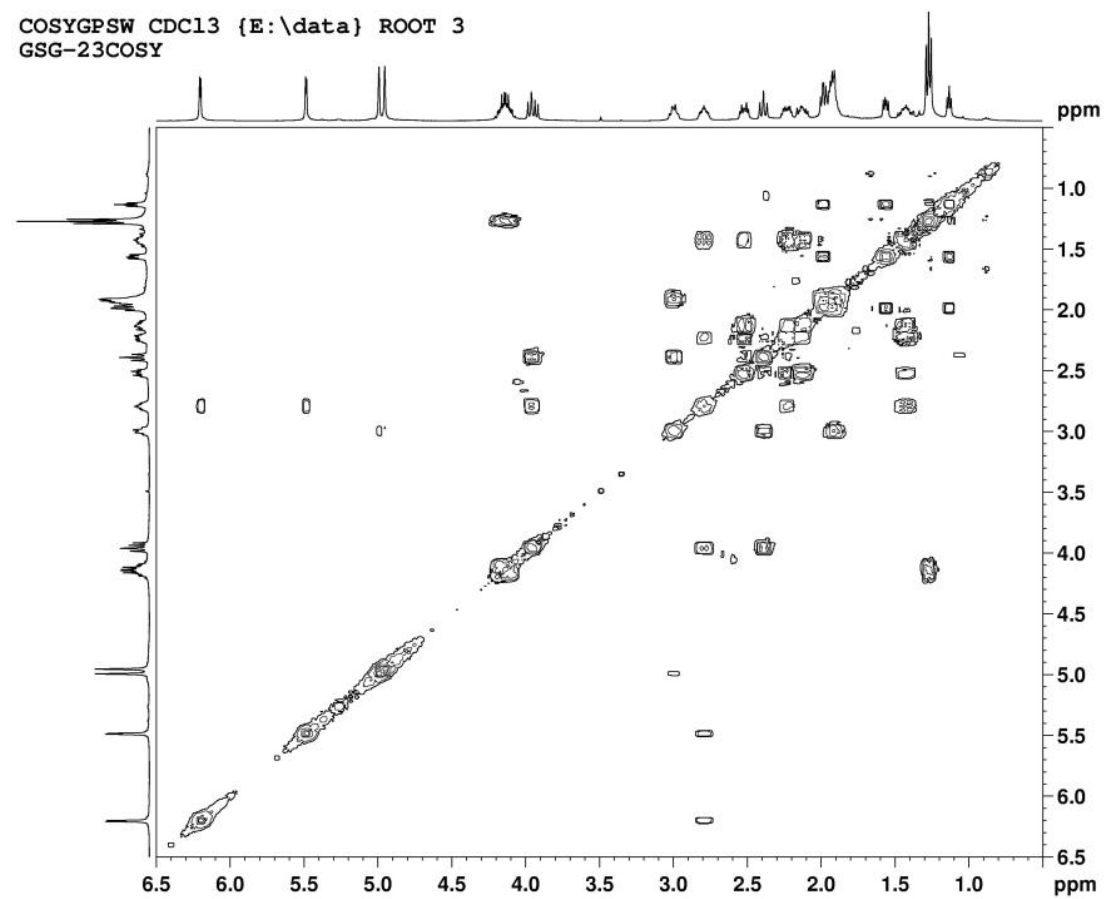

Figure S10. ${ }^{1} \mathrm{H}-{ }^{1} \mathrm{H}$ COSY Spectrum of $1\left(400 \mathrm{MHz}, \mathrm{CDCl}_{3}\right)$ 


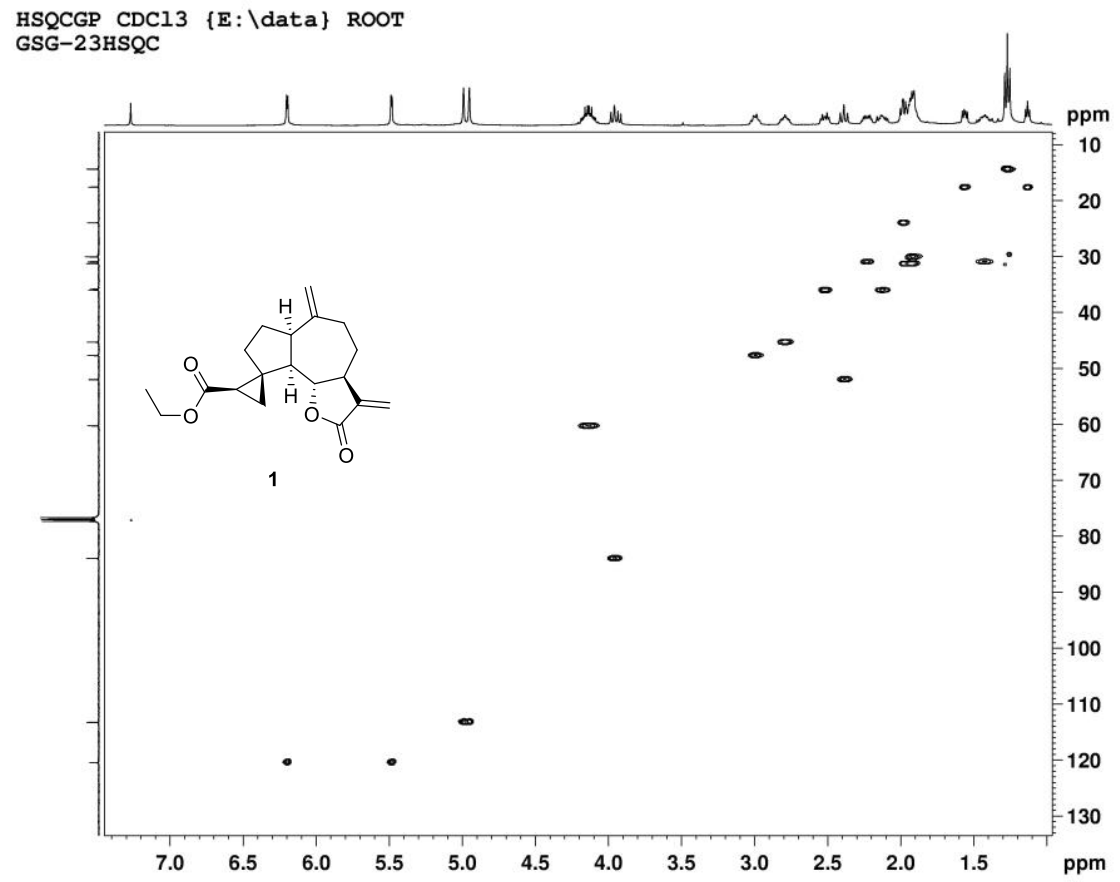

Figure S11. HSQC Spectrum of $1\left(400 \mathrm{MHz}\right.$ for $\left.{ }^{1} \mathrm{H} \mathrm{NMR}, \mathrm{CDCl}_{3}\right)$

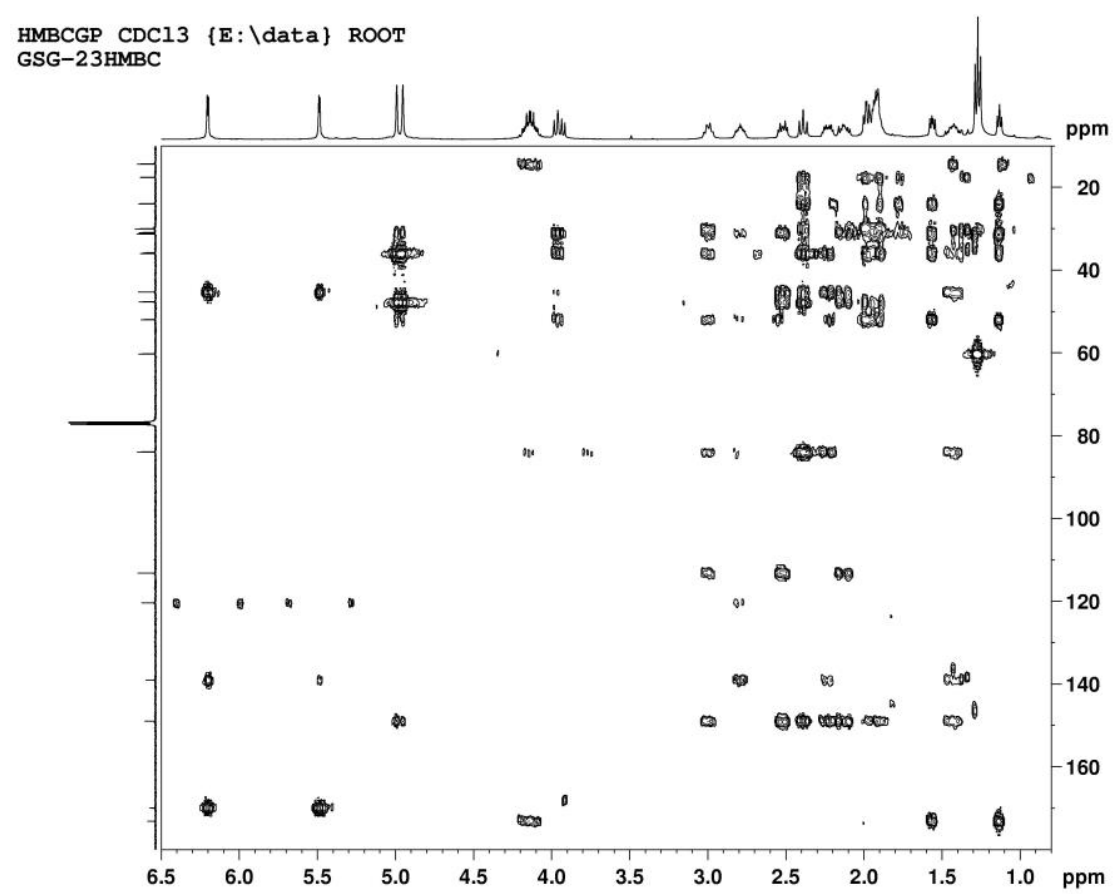

Figure S12. HMBC Spectrum of $1\left(400 \mathrm{MHz}\right.$ for $\left.{ }^{1} \mathrm{H} \mathrm{NMR}, \mathrm{CDCl}_{3}\right)$ 

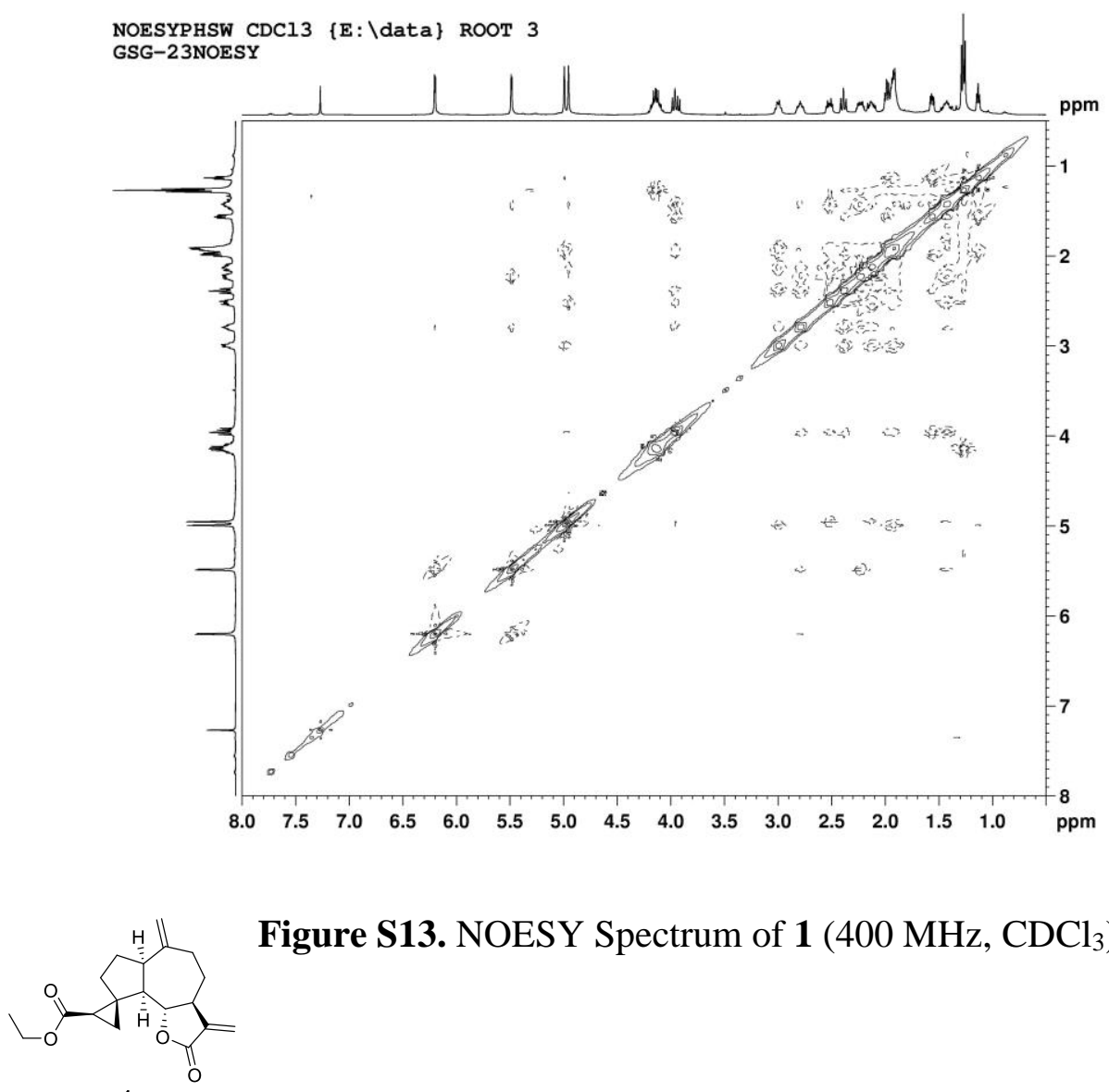

Figure S13. NOESY Spectrum of $1\left(400 \mathrm{MHz}, \mathrm{CDCl}_{3}\right)$

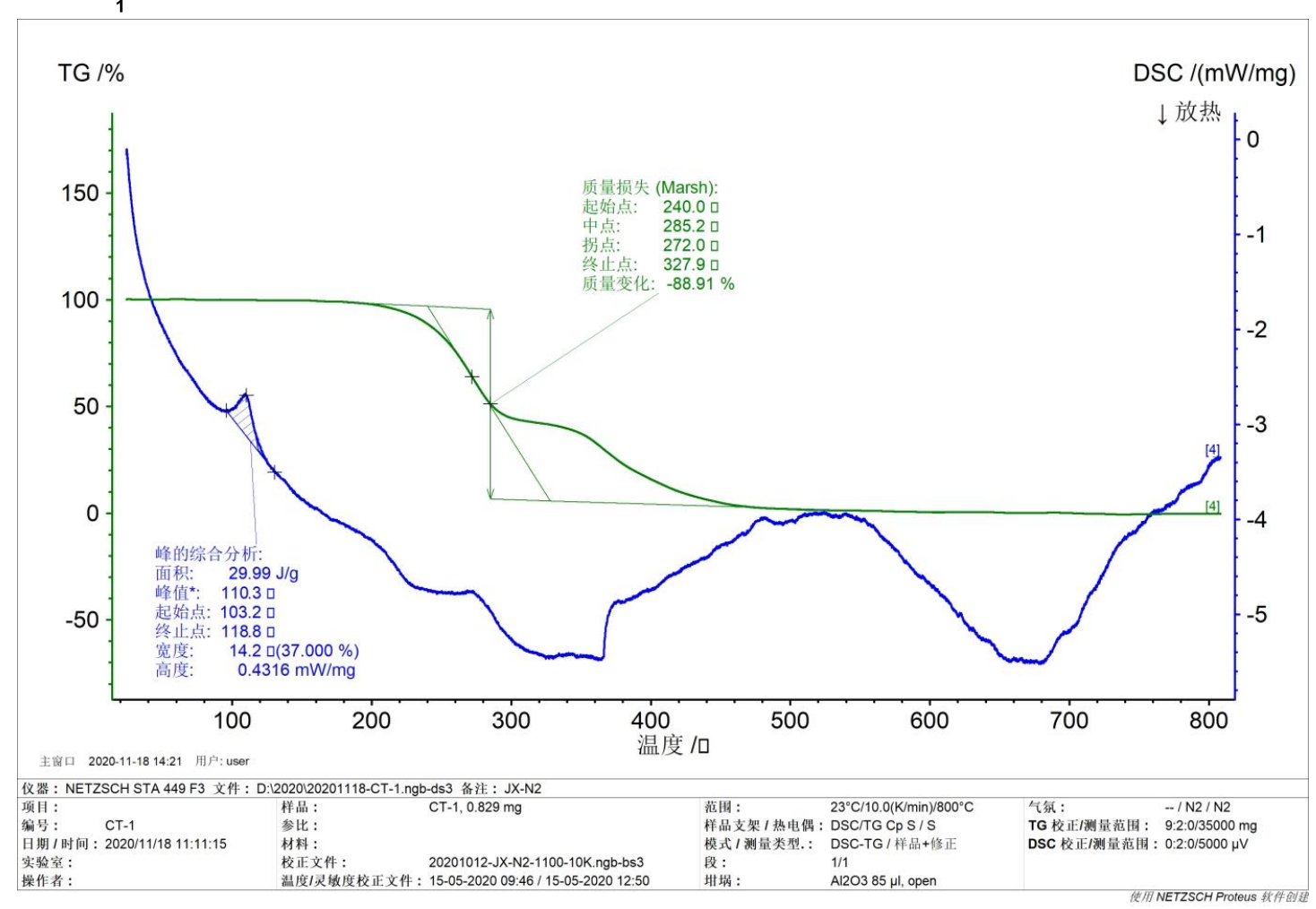

Figure S14. Melting point of 1 